by mainly hydrophobic side chains. The heavy atom obscures the rest of the ligand, which, however, is surmised to be oriented towards the surface. A number of side chains, including three serines, may be implicated in the saccharide binding site. In terms of the model of Edelman et al. the inositolbinding locus appears to be close to the slot housing the iodine, and too far therefore from the D-glucopyranose site to be identified with it. Edelman et al. maintain, because inositol is not, according to them, an inhibitor of the haemagglutination reaction, whereas their D-glucopyranose derivative suppresses both this and binding to polysaccharides, that the inositol binding mode is not functionally relevant. This may be, however, and the structure does not in any case at this stage throw any direct light on the physiological activity of concanavalin. It is generally supposed that the primary feature of its interaction with cells is its ability to cross-link receptor sites, by virtue of its stable dimeric, therefore divalent, structure. Its effect on mitogenesis is surmised to involve in the first place the promotion of cluster formation between the membrane glycoproteins: as it now seems clear that proteins are able to diffuse quite rapidly in the plane of the cell membrane, a bifunctional reagent like concanavalin will be expected to give rise to cross-linked oligomeric or polymeric networks. The possibility also exists of some secondary reaction with the cell surface following binding. Yahara and Edelman recently showed (ibid., 608) that concanavalin inhibits the coalescence of immunoglobulin on lymphocytes under the action of immunoglobulin-directed antibodies. The immunoglobulin-receptor complex is evidently free to diffuse in the membrane, and the anti-immunoglobulin antibody possesses again the ability to induce formation of a network. The structures that the concanavalin generates in the membrane evidently impede the motion of the immunoglobulin-receptor complex, or perhaps trap it by combining with it.

Along the same general lines, Shoham and Sachs (ibid., 2479) have shown that tissue-culture cells in different stages show differences in their response to fluorescein-conjugated concanavalin A. Amongst other effects that are found, transformed cells give a higher yield of fluorescence, and so also do normal cells after limited trypsin treatment. Time effects may be presumed to reflect the operation of a diffusion mechanism. It is not yet clear whether the differences depend on the availability of receptors, or, as seems to be the case for immunoglobulin receptors in erythrocytes, on differences in their capacity to form clusters.

FORENSIC SCIENCE

\section{Nuclear Detectives}

from a Correspondent

SOME of the most useful and perhaps surprisingly the least advertised benefits of nuclear technology have come from the application of activation analysis to forensic problems. The scientists and police officers from 30 countries who gathered in Glasgow from September 27-29 for the second International Conference on Forensic Activation Analysis reviewed the achievements (and occasional shortcomings) of the technique in topics as diverse as ballistics, toxicology, attribution of paintings and detection of counterfeit whisky.

Activation analysis, defined as the revelation of atomic composition through the modification of nuclear behaviour, is essentially a chemical technique involving the bombardment of the sample by neutrons (or, sometimes, other particles or radiations) followed by an assay of the induced radioactivity. When access to a nuclear reactor is available, the procedure is relatively simple and, for many elements, extraordinarily sensitive; for example the arsenic content of a one millimetre length of a single hair can readily be measured.

The forensic uses of activation analysis depend mainly on the detection and estimation of trace elements in material gathered from the scene of a crime or from a suspect. During the 1960s it was claimed that an individual could be identified by the trace element content of a hair. Some constituents of hair, such as sodium and zinc, show relatively little variation among individuals and are therefore not useful for identification. Other elements show considerable variation in concentration over a head, along a single hair, and it also varies according to diet and environment. Though some workers reported otherwise, R. Cornelis (Ghent) declared forthrightly that identification by activation analysis of hair is impossible.

Analysis of non-biological materials is less controversial. In some countries crimes involving shooting are routinely investigated by estimation of antimony and barium present in cartridge primers and on hands and clothing after firing. Study of such deposits around a bullet hole or wound allows the firing distance to be estimated. W. B. Renfro and W. A. Jester (Pennsylvania State University) showed how activation analysis of airborne residues allows the time elapsed since the firing of a shot to be estimated.

E. V. Sayre (Brookhaven National Laboratory) and colleagues from a number of laboratories and galleries in the United States described tests involving neutron bombardment of art work, followed by autoradiography, to reveal otherwise inaccessible details of materials and techniques serving to confirm or correct the attribution.

F. W. Lima (São Paulo) told of studies on counterfeit whisky. The bottles, labels, corks-and even the contents-were not distinguishable chemically from the genuine articles, but the lead foil caps were found, by anomalies in tin and antimony content, to be of local manufacture. The object of the well-planned counterfeiting was to sell Brazilian whisky (of its kind, quite good) as a genuine Scottish product at an inflated price.

\title{
Crystallization of Polyethylene
}

IN next Monday's Nature Physical Science evidence is presented to show that there are two distinct processes involved in the crystallization of polyethylene. D. C. Bassett, B. A. Khalifa and B. Turner of the J. J. Thomson Laboratory at the University of Reading report that the two processes dominate at low and high pressures, respectively, and by so doing they dispose of several suggested origins for the effect.

Crystallization of polyethylene at high pressures leads to crystals whose lengths along the chain direction are commensurate with the lengths of molecules. These crystals are known as extended chain crystals and are usually one or two orders of magnitude greater than the crystals obtained at atmospheric pressures.

The two distinct processes show up clearly in the work of Bassett and colleagues and it is shown that the change over from one form to the other occurs within a temperature range of only $5^{\circ} \mathrm{C}$ when a sample of polyethylene is crystallized at a pressure of $2.87 \mathrm{kbar}$, which is in between the high and low pressure limits.

Another feature of the work of the Reading team is that it is shown that the pressure at which one form of polyethylene changes into another form depends on the molecular weight of the polymer. But the unexpected result of this work is that the lower the molecular weight the higher the pressure required for the change to occur. Thus it turns out that it is easier to crystallize longer molecules into the very long chainextended crystals than it is to crystallize the shorter ones.

This experimental result is the exact opposite of the prediction of T. Kawai, whose explanation of the phenomena requires that the lower the molecule weight the greater should be the tendency to form longer crystals. 